\title{
LAS ESTRUCTURAS SOCIALES EN EL MARCO DE LA GLOBALIZACIÓN
}

\author{
FRANCISCO ENTRENA DURÁN
}

Universidad de Granada

PALABRAS ClaVe Adicionales

Autopoiesis, Dialéctica macro-micro, Global-local.

\author{
ADDITIONAL KEYWORDS \\ Autopoiesis, Dialectic macro-micro, \\ Global-local.
}

RESUMEN. Las estructuras sociales son vistas en este trabajo como realidades socialmente construidas, que están sujetas a la reflexividad y a la historicidad, cuya producción y reproducción se hallan cada vez más insertas en los procesos de globalización. Como consecuencia de esta situación, se experimenta una notable intensificación de la reflexividad de tales estructuras y, consiguientemente, un progresivo aumento de su grado de complejidad y de las dificultades para describirlas y explicarlas. Con el propósito de llevar a cabo dicha tarea desde una perspectiva holista, se proponen en el artículo unos criterios analíticos cuyo marco teórico pretende ser adecuado para el estudio de las relaciones de naturaleza dialéctica que se establecen, entre de una parte, la que se concibe como naturaleza reflexiva de las estructuras microsociales locales donde se desarrolla la vida cotidiana de la mayoría de la gente, y, de otra, la lógica, que aquí se considera como autopoiética o autoreferente, mediante la que se desenvuelven las estructuras macrosociales a escala global.

No por evidente conviene dejar de precisar que la estructura social no es la estructura de clases. El análisis de la segunda, que en ningún caso hay que confundir con la estratificación social, constituye sólo una parte fundamental para el estudio de la primera. Afirmar esto resulta necesario por cuanto que el concepto de clase, a pesar de que se ha visto envuelto en los últimos años en una especie de atmósfera enfermiza y decadente, sigue siendo una herramienta

Revista Internacional de Sociología (RIS)

Tercera Época, $n^{\circ}$ 27, Septiembre-Diciembre, 2000, pp. 125-150. 


\section{RIS}

útil para el estudio y la comprensión de las sociedades capitalistas avanzadas ${ }^{1}$. También, cuando se trata de analizar la estructura social, hay que examinar todos los elementos que, habitualmente, se incluyen en los estudios acerca de ella y que en un sentido amplio intervienen en su configuración. Así, se tiene que considerar la demografía, la estructura ocupacional y la división social del trabajo, el nivel de empleo, las desigualdades en el reparto de poderes y privilegios, la estratificación social, las identidades de clase o de status, la familia, la movilidad social, la distribución de la renta, la cantidad y la calidad de viviendas disponible, así como el sistema sanitario, la educación u otros servicios públicos, cuyo grado de desarrollo determina que existan unas cotas más o menos altas de bienestar social, etc..

No obstante, cuando en la actualidad se pretende llevar a cabo un análisis de alguna de las estructuras sociales concretas, además de tomar en cuenta la estructura de clases y los elementos referidos en el párrafo anterior, hay que tratar de describir y explicar los procesos de su producción y reproducción en el presente contexto de complejidad que origina la globalización; es decir, hablar de los procesos de producción y reproducción de las estructuras sociales desde una concepción de éstas como realidades socialmente construidas que están sujetas a la historicidad y a la reflexividad social. Como veremos, esta reflexividad se intensifica sobremanera como consecuencia de la creciente globalización en la que hoy se encuentran insertos tales procesos, a la vez que, a raíz de ello, se experimenta un progresivo aumento del grado de complejidad y de las dificultades de la tarea de describir y explicar las estructuras sociales. Con el propósito de que sirva de criterio de referencia para llevar a cabo esta complicada tarea, se propone aquí un modelo sistémico para el estudio de las estructuras sociales en el marco de la globalización basado en las tres dimensiones analíticas siguientes: la socioeconómica, la político-institucional y la simbólicolegitimadora. Este modelo posibilita el establecimiento de relaciones dialécticas entre la que se concibe como naturaleza reflexiva de las estructuras microsociales en las que se desarrolla la vida cotidiana de la mayoría de la gente, de una parte, y, de otra, la lógica, que aquí se considera como autopoiética o autoreferente, mediante la que se desenvuelven las estructuras macrosociales a escala global.

\footnotetext{
' Para llevar a cabo esta tarea, entre otras, es ineludible la consulta de la obra La estructura de clases en las sociedades avanzadas (1993). En ella, su autor, Anthony Giddens, efectúa una profunda y sugerente revisión de los presupuestos teórico-analíticos sobre los que originariamente fue construido por clásicos como Marx o Weber el concepto de clase, a la vez que trata de redefinir el mismo de acuerdo con las nuevas aportaciones sociológicas al respecto y en consonancia con las actuales circunstancias de las sociedades contemporáneas.
} 


\section{UNA APROXIMACIÓN CONCEPTUAL}

Dado el muy generalizado uso y la diversidad de significados que manifiestan en las ciencias sociales los términos globalización y estructura social, este primer epígrafe introductorio se dedica a revisar someramente los significados atribuidos a tales términos. La finalidad de ello es tratar de sentar las bases conceptuales para las argumentaciones posteriores. En primer lugar, por lo que se refiere a la palabra globalización, ha alcanzado gran difusión en las décadas finales del siglo XX; especialmente, durante la de los noventa. Sin embargo, el fenómeno social al que alude no es algo reciente, sino que viene de bastante atrás. Al fin y al cabo, un proceso de globalización fue el iniciado por la sociedad europea occidental cuando, a partir del Renacimiento, ésta empezó a difundir por el resto del globo terráqueo sus pautas de funcionamiento socioeconómico, de organización institucional y simbólico-culturales, consideradas durante siglos etnocéntricamente como el paradigma de la culminación del progreso y de la civilización humana. También, ya se estaba produciendo globalización cuando Cristóbal Colón se embarcó en un viaje que inintencionadamente le llevó a descubrir el territorio que luego acabó siendo denominado como América. Incluso, adoptando una perspectiva menos centrada en la realidad europea, ya fueron formas de globalización las exploraciones y la expansión del comercio de los chinos que tuvieron lugar en el siglo $\mathrm{X}$, en tanto que fueron fenómenos que trascendieron el marco socioeconómico, institucional y cultural relativamente local o regional en el que se originaron $y / o$ al que afectaron o, por lo menos, llegaron a extensiones mayores del planeta que el ámbito territorial del que partieron ${ }^{2}$.

Posteriormente, la conquista y la colonización del centro y del sur de América, llevada a cabo por España y Portugal, y la de Norteamérica, por parte de Gran Bretaña y Francia, son ejemplos de la gradual propagación de los paradigmas socioeconómicos, político-institucionales y simbólico-culturales del orden social occidental por todo el mundo, es decir, de globalización. Modalidades de globalización fueron también las diversas colonizaciones efectuadas, durante el siglo XIX y principios del XX, por un reducido número de Estados europeos que extendieron su dominación abarcando virtualmente la totalidad del continente africano, junto con extensas áreas de Asia, Latinoamérica y Oceanía.

Años después, tanto las teorías de la modernización como las perspectivas críticas elaboradas como reacción frente a ellas (teoría de la dependencia, teoría del intercambio desigual, o teoría del centro-periferia), también hacían referencia

${ }^{2}$ Me sugiere esta apreciación la lectura de Alessandro Bonanno (1994: 17 y ss.). 
RIS

a procesos de globalización. Unas y otras seguían concibiendo la modernización como una tácita continuación de los procesos de globalización que habían sido iniciados casi cinco siglos antes a raíz de la gradual expansión del modelo de orden social occidental por el resto del mundo.

Pero, el término globalización, cuyo uso se ha extendido tanto a partir de la última década del siglo XX, ya no hace referencia a un proceso de occidentalización del mundo, sino que alude a una situación completamente nueva en la que Occidente ya no es el patrón indiscutible de referencia a seguir por todos los que aspiran a desarrollarse y a mejorar socialmente. En esta situación, se está experimentando una creciente internacionalización e intensificación del número y del ritmo de la circulación de personas, ideas y mercancías, así como de los flujos socioeconómicos, institucionales y culturales, cuya influencia y desarrollo se incrementan día a día en todas las direcciones del planeta y a escala global.

Como consecuencia de los actuales procesos de globalización, estamos entrando en una época en la que es la humanidad, como una totalidad, la que constituye la unidad social determinante, el modelo de lo que entendemos por sociedad y, por lo tanto, la base de referencia de muchos estudios científicos. La humanidad está inmersa en un proceso de integración global. Las sociedades se hacen cada vez más interdependientes e interconectadas. La humanidad no es ya un simple agregado estadístico, o una categoría filosófica; se ha convertido en una entidad social real, en una totalidad que abarca a toda la población mundial (Sztompka: 1995, 111). En estas circunstancias, se está experimentando una transición hacia formas de organización más complejas de alcance global, con la consiguiente pérdida de soberanía y de capacidad de actuación socioeconómica de los Estados individuales, debido a que, en el nuevo sistema mundial, muchas decisiones importantes son progresivamente tomadas en escenarios transnacionales. Hoy ya podemos hablar de la existencia de un único sistema mundial, lo que no ha sucedido así ni siquiera en el pasado más reciente, pues como señala Peter Worsley (1984: 1), hasta nuestros días no existió nunca una sola sociedad humana. La unidad social que sirve hoy de punto de referencia para muchos fenómenos de desarrollo y cambios estructurales no está ya constituida por Estados particulares, sino por la humanidad dividida en asociaciones de Estados (Elias, 1990: 188 y ss.).

Desde el punto de vista de lo que constituye el objeto de estudio de este trabajo, interesa destacar como, en estas condiciones, la vida cotidiana de las personas que tiene lugar en el seno de las estructuras sociales locales concretas está hoy más conectada a lo que sucede a escala global que nunca antes $\mathrm{y}$, por consiguiente, afectada por ello en un grado tal que era imposible e inimaginable en el seno de las relativamente autárquicas y autosuficientes, en sus procesos de producción y reproducción social, estructuras sociales locales de un pasado todavía no muy lejano ${ }^{3}$. 
Posteriormente, se entrará más a fondo en la cuestión de cómo afecta la globalización a la estructura social. Por ahora, de acuerdo con el propósito para esta introducción antes referido, concentraremos nuestra atención en lo que el concepto de estructura social ha significado y significa en la Sociología. En este sentido, hay que tener presente que dicho concepto tiene una larga tradición en la evolución del pensamiento sociológico, especialmente a partir de Marx, Spencer y Durkheim. Sobre todo, su utilización alcanzó una extensión muy notoria como consecuencia del considerable desarrollo experimentado por la corriente estructural-funcionalista de la Sociología norteamericana después de la Segunda Guerra Mundial y, también, a resultas de la extensión e influencia que alcanzó el estructuralismo francés en las décadas de los sesenta y setenta ${ }^{4}$. En aquellas circunstancias, preocupado por la excesiva difusión y polisemia del término estructura, el antropólogo norteamericano Kroeber llegó a calificar su uso como una "concesión a la moda" que no añadía absolutamente nada a lo que ya se sabía antes de emplearlo, salvo "una agradable sensación al oído" (Levi-Strauss, 1958: 304).

Si bien es cierto que a la imprecisión conceptual de este término ha contribuido su excesiva generalización a ámbitos teórico-prácticos muy diversos, también es verdad que su notable expansión no responde sólo al capricho o al afán de causar buen efecto por parte de quienes lo emplean. En concreto, por lo que se refiere al caso específico de la Sociología, la utilización del término estructura suele estar justificada por el propósito de aplicar una metodología que implica una forma sistémica de enfrentarse al análisis de la realidad social, considerando a la misma como una totalidad de partes interdependientes, como una estructura con unas leyes de interrelación y de organización entre sus respectivos elementos ${ }^{5}$.

\footnotetext{
${ }^{3}$ Digo relativamente porque, en realidad, la autarquía no ha existido nunca en su estado puro. Sin embargo, puede considerarse que al ideal de la autarquía se aproximaban más las estructuras sociales del mundo tradicional, cuyo ámbito vital cotidiano era, por lo habitual, un microcosmos relativamente cerrado y circunscrito a un espacio territorial, más o menos autosuficiente, que tendía a bastarse a sí mismo en el plano de su producción y reproducción socioeconómica, político-institucional y simbólico-cultural.

${ }^{4}$ Levi-Strauss y Lacan son quizá los más importantes precedentes inmediatos del estructuralismo. Sin embargo, unas fuentes más remotas de esta corriente intelectual están en el método empleado para el estudio de la Lengua por el profesor Ferdinand de Saussure. Saussure murió en 1913 tras una larga enfermedad. En 1915, Charles Sally, Albert Sechehaye y Albert Riedlinger, que habían sido alumnos y asiduos oyentes suyos, publicaron el Curso de Lingüistica General, basándose para ello en las notas tomadas en clase. En esta obra se contienen las líneas generales del pensamiento y de la metodología saussurianas (Millet y Varin d'Aivelle, 1975: 12 y 13).

${ }^{5}$ De este modo, "al estudiar la estructura estudiamos esencialmente la interrelación o disposición de partes de alguna entidad total, de algún todo... el adjetivo social especifica el carácter de ese 'todo', el cual es la sociedad o alguna de sus subdivisiones ...."(Nadel, 1966: 31).
} 


\section{RIS}

De entre los muy diversos significados que le son asignados o atribuibles, en el ámbito de la Sociología pueden destacarse dos modos diferenciados de entender la estructura social: a) el que tiende a identificarla con la estructura de la realidad empírica a partir de la que se produce y reproduce el orden social, y el ideal del mismo, existente en la colectividad que se estudia; y, b) la perspectiva de aquellos otros que, en cambio, consideran la estructura como un mero modelo.

En la primera forma de concebir el término estructura se podría incluir el análisis estructural-funcionalista. Así, éste tendía a centrarse, preferentemente, en el estudio del funcionamiento y la finalidad ideal de los marcos institucionales y simbólico normativos empíricos, sobre los cuales se encarna la ideología o saber explícito que las sociedades tienen de sí mismas ${ }^{6}$. A diferencia de este punto de vista, otros autores han concebido la estructura social como una mera construcción lógica de la mente (Leach, citado por Nadel, 1966: 226) o como un puro modelo instrumental en manos del investigador que es preciso distinguir de la realidad empírica a partir de la que se construye (Levi-Strauss, 1958) ${ }^{7}$.

\section{HISTORICIDAD Y REFLEXIVIDAD DE LA ESTRUCTURA SOCIAL}

Por mi parte, concibo la estructura como un modelo socialmente construido y con ello me refiero, tanto a la construcción social que supone el modelo conceptual mediante el que el investigador trata de describir y explicar la estructura de la más o menos compleja y cambiante maraña de relaciones existente en cualquier sociedad, como a la realidad de la producción y reproducción social de esa estructura, en tanto que modelo de articulación de un orden social

\footnotetext{
'Como escribió Jesús Ibáñez (1979: 21), todo "orden social produce representaciones, saberes inmediatos, de sí mismo. Estos saberes funcionan como matrices de discursos, como textos implícitos que producen discursos explícitos, discursos que hacen tolerable ese orden haciéndolo comprensible, produciendo una explicación global y compatible de todos los fenómenos que ese orden regula, una explicación que da razón de esos fenómenos".

${ }^{7}$ Según Levi-Strauss (1958: 305-306), los requisitos que tiene que cumplir dicho modelo para merecer el nombre de estructura son cuatro: 1) una estructura tiene un carácter de sistema. Consiste en unos elementos tales que una modificación en uno de ellos conlleva una modificación de todos los demás; 2) todo modelo corresponde a un grupo de transformaciones, de las que cada una pertenece a un modelo de la misma familia, si bien el conjunto de estas transformaciones constituye un grupo de modelos; 3 ) las propiedades indicadas en los puntos anteriores permiten prever de qué forma reaccionará el modelo en caso de modificación de uno de sus elementos; 4) el modelo ha de ser construido de tal modo que su funcionamiento pueda dar cuenta de la totalidad de los hechos observados.
} 
determinado. Evidentemente, el objetivo a conseguir es que el modelo teórico del investigador se acerque lo más posible al de la realidad de la estructura social que trata de describir y explicar. Sin embargo, el hecho es que ese objetivo nunca es plenamente alcanzado, sobre todo debido a la inconmensurabilidad de cualquier estructura social - $y$, especialmente, la de las altamente complejas estructuras sociales del globalizado mundo actual. Por este motivo, es fundamental distinguir entre la estructura social y las herramientas conceptuales o modelos que se emplean para su análisis, entre las limitaciones de la mera utilidad instrumental de las provisionales construcciones teórico-metodológicas que son esos modelos y la, nunca completamente conocida, dinámica multivocidad de la totalidad social. Ante la inconmensurabilidad de cualquier estructura social, la única alternativa sensata es adoptar una posición analítica que reconozca las limitaciones de nuestros modelos teóricos para el conocimiento de la misma, los cuales sólo muestran una visión, más o menos esquematizada y simplificada, de la totalidad multívoca e inabarcable de su dinámica de funcionamiento y de cambio. Utilizando palabras de Jesús Ibáñez (1979: 210), podemos considerar la estructura como un "punto de vista del receptor" - el investigador de lo social en nuestro caso-, con respecto a una totalidad social en perpetuo cambio, cuya complejidad no puede ser completamente acotada por las, necesariamente restringidas, estrategias metodológico-conceptuales que para ello empleamos.

Pero, que el modelo analítico de estructura social adoptado en un determinado caso refleje el "punto de vista" al respecto del investigador, no significa que éste pueda construir como arbitrariamente se le antoje dicho modelo, cuya validez depende de que el "punto de vista" que lo subyace sea adecuado para contribuir a conformar, junto con otros "puntos de vista", un horizonte de sentido socialmente significativo (Ricoeur, 1969: 53). En otras palabras, aun cuando un modelo teórico de estructura social nunca podrá sustituir a la realidad social que trata de representar, por lo menos ha de ser adecuado para llevar a cabo de la mejor manera posible el objetivo más arriba mencionado de describirla y explicarla. De no ser así, el modelo pierde su condición de tal y desaparece la relación mutuamente reflexiva entre el quehacer del sociólogo y la sociedad (Pérez de Guzmán, 1995: 180). Dicha relación hace posible que la tarea del primero tenga razón de ser y sea socialmente fructífera, al contribuir a la conformación de ese horizonte de sentido socialmente significativo antes referido.

Como construcción social que es, la estructura social está sujeta al cambio y por tanto a la historicidad y a la reflexividad social. Pero, veamos primero que queremos decir con la expresión historicidad de la estructura social. En relación con esto, de manera más o menos consciente, las visiones totalitarias o absolutistas de la estructura social suelen sustentarse en una imagen arquitectónica de la misma que las incita a presuponer que ésta es una construcción integrada 
por individuos que se comportan como si fueran piezas o elementos inertes. De hecho, como dice Peter L. Berger (1989: 253), la generalidad de los sociólogos teóricos utilizan, a menudo, el lenguaje de los arquitectos. Así, hablan de "edificios" o "construcciones" teóricas y describen su tarea como "edificación de teorías". Sin embargo, el propio Berger nos recuerda que en el edificio social faltan piezas, que no está terminado. Específicamente, en lo que se refiere a la estructura social, esto significa que la misma no es algo acabado y definitivo, sino una construcción social inconclusa que está sometida a continuos procesos de construcción, deconstrucción y reconstrucción a lo largo de la historia; de ahí, su carácter circunstancial y cambiante, sin que ello sea óbice para que, como sostiene Nisbet (1975: 294 y ss.), puedan observarse en la estructura de cualquier orden social una serie de persistencias que permanecen a lo largo del transcurrir del tiempo histórico.

Entre estas persistencias, cabe incluir las pautas de relación y de organización de estructuras sociales, en tanto que pautas que, cuando se dan las condiciones de lo que podría considerarse como una cierta continuidad en el transcurso del devenir histórico de los órdenes sociales, suelen manifestar un apreciable grado de perdurabilidad en sus procesos de producción y reproducción. En cualquier caso, el hecho de tomar en cuenta estas permanencias no es óbice para que podamos albergar también una concepción dinámica de las estructuras como realidades cambiantes cuya génesis y evolución se gestan en procesos observables a lo largo de la historia. Es más, la consideración de la naturaleza procesual e histórica de las sociedades y de las estructuras desarrolladas por ellas es un hecho ineludible en cualquier investigación social. Por ello, cuando un estudioso se limita a centrarse en el instante presente de la vida de una sociedad, es víctima de una ilusión, puesto que todo es historia. Lo que sucedió ayer es historia, y lo que ha sido dicho sólo un minuto antes también es historia. Pero, sobre todo, el investigador que así actúa se condena a no conocer este presente, ya que solamente el hecho de tomar en cuenta los procesos históricos permite sopesar, y evaluar en sus relaciones respectivas, los elementos del presente (Levi-Strauss, 1958:17).

La estructura del orden social vigente en un momento histórico dado no es en sí la traducción estructurada de la misma sociedad, tal como solían pretender los estructural-funcionalistas, sino sólo una de las muchas formas posibles de articulación de ese orden en sus propósitos de regulación (socioeconómica, político-institucional y simbólico-cultural) y de búsqueda individual y colectiva de sentido y de totalidad. Ante una realidad social continuamente cambiante, toda estructura afirma simbólicamente su pretensión de durar, solventando así en lo práctico y en lo simbólico el miedo de sus sujetos humanos integrantes al azar, a la contingencia y a la muerte. Dicha pretensión se realiza a través de la creación de instituciones y de sistemas pautados de relaciones, cuyas estructuras son construcciones sociales que constituyen el substrato sobre el que cristalizan 
los valores y las normas que fundamentan el orden social. Mediante éste, a su vez, las colectividades humanas tratan de cumplir las funciones sociales encaminadas a materializar y a concretar históricamente su aspiración a perpetuarse en el tiempo.

No obstante, una cosa son las funciones que manifiestamente se propone cumplir una estructura social concreta y otra muy distinta son las funciones que realmente esa estructura cumple. Como ya señalara Merton (1970), frente a lo que presuponían las tesis estructural-funcionalistas clásicas, lo que verdaderamente define un orden social cualquiera no son las funciones que trata de cumplir sino sus disfunciones; es decir, lo que se podría formular como la distancia entre sus fines sistémicos y/o aspiraciones ideales colectivas de identidad y cierre como totalidad $^{8}$, de una parte, $y$, de otra, el papel real que desempeñan las instituciones, pautas socioeconómicas y saberes simbólico-culturales explícitos que se han propuesto como cauces para conseguir estos fines en un momento determinado de la historia. Como se ha dicho antes, esta distancia se produce, sobre todo, porque toda acción social humana tiene efectos no deseados o imprevistos, los cuales, a su vez, son debidos a que los individuos no actúan mecánicamente en el seno de las estructuras sociales ejecutando las funciones para las que han sido socializados como si fueran autómatas, como si desempeñaran literalmente las funciones de un programa informático para el que han sido codificados. Contrariamente a esto, la acción social de los sujetos humanos individuales y colectivos es reflexiva; es decir, en mayor o menor grado, tales sujetos tienden a debatir, replantearse o reflexionar continuamente acerca de su visión de las estructuras sociales en las que se hallan insertos o de las que depende su posición social, así como dicha posición?.

Todas las sociedades de cualquier tiempo y lugar han sido y son reflexivas. Sin embargo, en las estructuras de las, relativamente más circunscritas a lo local que ahora, sociedades del pasado existía un bajo efecto de la reflexividad social, en comparación con lo que sucede actualmente. En ellas los procesos económicos, sociales e institucionales eran más fácilmente controlables o

\footnotetext{
${ }^{8}$ En todo orden discursivo o social hay una aspiración de cierre y de universalización que se manifiesta en su tendencia a constituirse como estructura cerrada. Jesús Ibáñez dice: "el discurso 'estructural' tiende a construir un texto que cierra la historia: excluyendo las posibilidades que quedan fuera, incluyendo las posibilidades que encierra en una jerarquía positiva" (1979: 210).

${ }^{9}$ Como dice Emilio Lamo de Espinosa, "los hombres tienen la perniciosa habilidad de ser inteligentes, es decir, poseen la doble capacidad de pensar sobre sí mismos y su situación (i.e. de producir etnociencia) y de aprender lo que sobre ellos y sus situaciones dicen otros (i.e. de hablar y leer)" (1990: 166).
} 


\section{RIS}

previsibles porque se desarrollaban dentro del ámbito espacial local, regional o del Estado-nación. Este ámbito funcionaba como instancia aglutinadora de la sociedad, que era percibida de manera más o menos unificada y monolítica, como si se tratara de un tejido, una telaraña. Nunca hubo, realmente, una sociedad tan perfectamente articulada, unificada y estructurada que funcionara de acuerdo con lo que se sugiere en esta metáfora. Sin embargo, esta imagen de la estructura de la sociedad estaba, de modo más o menos explícito, presente en los grandes modelos teóricos construidos por los clásicos de la teoría sociológica del siglo XIX ${ }^{10}$.

No obstante, cuando las sociedades han tenido unos niveles de equilibrio y de regulación social relativamente más altos que los del vertiginoso y globalizado mundo actual, ha podido resultar relativamente más fácil aventurarse a elaborar modelos acerca de su estructura social, entre otras razones porque ésta era más estable y previsible en su funcionamiento. Se explica, así, que, por ejemplo, un enfoque sistémico de la estructura social como el propuesto por Parsons en su obra El sistema social (1976) encontrara un terreno propicio para su plausibilidad y difusión en los años en los que en las sociedades desarrolladas estaban en sus cotas más altas la previsión social y la estabilidad inherentes al Estado keynesiano de bienestar.

\section{REFLEXIVIDAD Y AUMENTO DE LA COMPLEJIDAD}

En contraposición a la situación antes referida, en las estructuras de las sociedades avanzadas actuales se produce una intensificación de la reflexividad que está estrechamente relacionada con el hecho de que en ellas tenga lugar una constante reestructuración de sus pautas socioeconómicas, político-institucionales y simbólico-culturales como consecuencia de su creciente globalización. La circunstancia de que ésta conlleve una elevada circulación de personas, ideas y mercancías, a escala mundial, da lugar a que los conceptos en torno a la sociedad, las preferencias y las expectativas de mucha gente o de diversos colectivos

\footnotetext{
${ }^{10}$ Fundamental en la imaginación del siglo XIX era la concepción de la sociedad como un tejido - $\mathrm{y}$, en las alucinaciones literarias, una telaraña. $\mathrm{O}$, en el espíritu filosófico más abstracto, tal como lo elaboró Hegel, cada cultura, cada «período» de la historia, y -correspondientementecada sociedad, era una totalidad estructuralmente entrelazada, unificada por algún principio interno. Para Hegel, era el Geist o espíritu interior. Para Marx, era el modo de producción, que determinaba todas las relaciones sociales" (Bell, 1977: 21).
} 
poblacionales locales, regionales, de clase o de status se estén rearticulando y reformulando continuamente, a la vez que presentan manifestaciones muy diferentes, bastante inestables y, con frecuencia, opuestas entre sí. La gran multiplicación y aceleración de los flujos económicos, sociales y culturales que se deriva de la globalización origina unas crecientes dificultades para regular, analizar y comprender unas estructuras sociales sujetas a tan acelerados, complejos e imprevisibles ritmos de cambio.

Cada vez se está más lejos de aquellas situaciones en las que las estructuras sociales se producían y reproducían durante milenios en unas condiciones en las que el «cuando» y el «donde» (es decir, el tiempo y el espacio) solían desarrollarse unidos y vinculados a unos ámbitos territoriales locales específicos. Contrariamente a este «localismo», hoy las estructuras sociales se gestan y desarrollan, cada vez más, en relación y simultaneidad con otras estructuras sociales, las cuales, con frecuencia, se desenvuelven en ámbitos espaciales muy distantes y diferentes sociocultural, económica e institucionalmente. Esto propicia lo que podría considerarse como una especie de globalización u «homogeneización» mundial de los ritmos temporales de producción y reproducción social de las estructuras sociales, que contrasta claramente con la diversidad que han manifestado estos ritmos en el pasado durante los milenios de existencia de las distintas sociedades humanas en los que éstas estaban mucho más cerca de la autarquía socioeconómica, cultural y político-administrativa que en el globalizado mundo actual, cada vez más interconectado a escala planetaria.

Uno de los efectos más significativos de todo esto es una paulatina devaluación de los presupuestos unilineales ascendentes sobre los que durante mucho tiempo pretendió explicarse la dinámica de cambio de las estructuras a lo largo de la historia. Presupuestos, según los cuales podía establecerse, más o menos claramente, una secuencia evolutiva entre los diferentes estadios de desarrollo que iba adquiriendo una determinada estructura social ubicada en un ámbito espacial determinado a lo largo de los sucesivos momentos temporales en los que era observada. La generalizada confianza en la idea de progreso asociada a dicha secuencia evolutiva con relación a Occidente, manifestada durante largo tiempo por la sociedad y las Ciencias Sociales, aparte de deberse a que han estado muy extendidos hasta hace poco los efectos de aquellos momentos iniciales en los que una considerable parte de la humanidad vivía al socaire del entusiasmo por los indudables adelantos del desarrollo científico-tecnológico y a que consecuentemente no se hayan generalizado ni la conciencia ni los efectos negativos o «consecuencias perversas» de la modernidad (Giddens, 1999; Beriain, 1996), en cierta medida, ha sido posible porque en las, relativamente más autárquicas (a escala regional o estatal), sociedades pasadas era más fácil que ahora corroborar dicha idea mediante la comparación de su entonces presente con su pasado, ya que en ellas se disponía de unas referencias espacio 
RIS

REVISTA INTERNACIONAL DE SOCIOLOGLA

№ 27. Septiembre - Diciembre, 2000

FRANCISCO ENTRENA IURÁN

temporales con respecto a sus estructuras sociales que eran más fácilmente delimitables, en relación con las dificultades que a este respecto se plantean en las crecientemente conectadas a lo global estructuras sociales actuales. Estas referencias hoy han dejado de ser tan claras, entre otros motivos, por las razones siguientes: a) la pérdida de la centralidad paradigmática de Occidente - es decir, el eurocentrismo tiene cada vez menos cabida-, lo que hace que ya no esté claro hacia donde camina o ha de caminar el mundo y por consiguiente cual es la dirección que ha de adoptar el proceso de cambio de una determinada estructura social; b) el hecho de que, al estar conectados simultáneamente a escala planetaria los procesos de producción y reproducción social de las estructuras, disminuyan para los actores sociales en ellas insertos las posibilidades de intervención y de regulación social con relación a las mismas y, por consiguiente, las oportunidades de incidir en la planificación y en el rumbo de su cambio social; c) la creciente complejidad y dificultades para describir y explicar tales procesos que de todo ello se derivan.

La globalización se manifiesta y materializa, sobre todo, como globalización de lo local. En otros términos, el hecho de que la vida cotidiana de la mayoría de la gente se desarrolle en el seno de estructuras sociales crecientemente globalizadas no significa que esa vida tenga lugar en escenarios de dimensiones planetarias. La mayor parte de la población sigue viviendo, como en el pasado, en el marco de estructuras sociales que constituyen ámbitos microsociales específicos fácilmente localizables e identificables. En casi todos los casos, esos ámbitos se desarrollan en un espacio geográfico o físico determinado que es también su espacio social o escenario en el que tiene lugar su acción social cotidiana. Lo que sucede es que, en las actuales condiciones de la modernidad tardía, vivimos en el mundo en un sentido y forma diferentes de las anteriores eras de la historia. La mayoría de la gente continúa todavía llevando una vida local situada contextualmente en un tiempo y en un espacio. Sin embargo, cada vez es mayor la penetración de influencias lejanas en las estructuras sociales de los entornos locales, lo que significa que esas estructuras y la experiencia social de los individuos que en ellas viven están verdaderamente globalizadas (Giddens, 1991: 187 y ss.).

No obstante, si bien como he dicho la generalidad de las personas desarrolla su cotidianeidad en el seno de ámbitos espaciales locales específicos, existe una minoría, que tiende a incrementarse como consecuencia de la globalización, cuya vida diaria transcurre en continuos viajes a lo largo del mundo (por ejemplo, los ejecutivos de las compañías transnacionales o los miembros de organizaciones de alcance mundial como la ONU o la UNESCO). Para esta minoría, suele ser habitual que su espacio social —es decir, el círculo de sus relaciones socialesexceda los límites de su lugar geográfico de residencia. Pero, incluso en esta situación, puede afirmarse que la vida cotidiana de estas personas se desarrolla en el ámbito de estructuras sociales cuyas redes de relaciones personales son 
localizables y más o menos restringidas e identificables, aunque sea sólo en el plano social. También, son claramente localizables en lo social las redes relacionales de carácter virtual establecidas a través de la conexión a internet que permiten el contacto regular de personas muy distantes entre sí.

En definitiva, aunque los procesos de producción y reproducción de las estructuras sociales siguen teniendo lugar en su mayor parte a escala relativamente local —en el marco de determinadas regiones o de Estados específicos-, tales procesos cada vez se encuentran más insertos en la presente dinámica de la globalización y, por tanto, cada vez más afectados por ella. Cualquier manifestación de la estructura social es una realidad especialmente compleja en la que puede contemplarse una enorme variedad de fenómenos sociales que experimentan una.continua interacción y una constante mutación. Pero, esta complejidad se acrecienta especialmente en el presente contexto, sobre todo, debido a que la cada vez mayor globalización de las estructuras sociales hace que en éstas tenga lugar esa fuerte intensificación de la reflexividad de la que se trataba más arriba.

\section{UN ANÁLISIS SISTÉMICO}

Con el fin de tratar de racionalizar la complejidad de las estructuras sociales antes referida, se propone a continuación un modelo sistémico basado en una concepción de las mismas como totalidades cuyas dimensiones socioeconómica, político-institucional y simbólico-cultural están relacionadas entre sí. Las estructuras son consideradas en dicho modelo como dialécticamente determinantes/determinadas de/por los sujetos sociales concretos que forman parte de ellas. Éstos actúan reflexivamente, lo que significa que «se comportan creativamente» según las específicas circunstancias en las que se encuentran en cada caso, y no ateniéndose al seguimiento más o menos literal de unos códigos o paradigmas abstractos y externos a ellos inscritos en las estructuras socioeconómicas o culturales, tal y como, respectivamente, solían suponer las corrientes de pensamiento marxistas y las estructural-funcionalistas, dados sus enfoques más o menos deterministas de la estructura social. Contrariamente a este punto de vista, como seres reflexivos que son, los sujetos humanos (individuales y colectivos) son parte activa de la estructura social en la que se desenvuelven, a cuya producción, reproducción o modificación contribuyen, aunque, por lo general, con resultados muy diferentes de sus propósitos e intenciones conscientes. Dicho de otro modo, el hecho de considerar la reflexividad de las estructuras sociales y de los sujetos humanos en ellas inmersos implica no sólo asumir que tales sujetos no son marionetas de esas estructuras, sino también negar que éstas o su evolución histórica sean el resultado de lo que los humanos quieren o se proponen conseguir mediante sus acciones. Frente a esta presupo- 
RIS

REVISTA INTERNACIONAL DE SOCIOLOGIA

No 27, Septiembre - Diciembre, 2000

sición voluntarista, tan profundamente arraigada en el antropocentrismo moderno $^{11}$, hoy hemos llegado a constatar, como consecuencia de nuestra experiencia intelectual y vital, esos efectos no deseados o imprevistos de la acción social humana a los que se hacía referencia antes, de tal forma que lo que acontece suele tener muy poco que ver con lo que distintos sujetos individuales y colectivos han pretendido o intentado conseguir. Esto, en muy gran medida, como consecuencia de la propia reflexividad de tales sujetos.

En el presupuesto de la existencia de la reflexividad, tal y como ésta es aquí concebida, se sustenta el modelo sistémico que propongo seguidamente para analizar las estructuras sociales en el contexto de la globalización. En dicho modelo, que se esquematiza en el tabla 1, se integran los aspectos macro y microsociales, así como tres dimensiones objetivas y subjetivas de la estructura social. Estas tres dimensiones actúan de manera interrelacionada e interdependiente como los elementos de un sistema. Con este sistema teóricoanalítico trato de modelizar el marco global que constituye el escenario y la circunstancia condicionante de la producción y reproducción de las complejas estructuras sociales concretas en el presente contexto de mundialización. En dicha tabla hago sólo una relación general no exhaustiva de los procesos que han de ser tomados en cuenta cuando se acomete el análisis de cualquiera de esas estructuras. Dejo al lector la decisión de aumentar o reducir en cada caso la relación que propongo. De cualquier modo, la mayor o menor relevancia conferida a cada uno de tales procesos, sus características y el número de ellos a

\footnotetext{
${ }^{11}$ En este sentido, tanto el marxismo como el estructural-funcionalismo, no obstante su énfasis en la capacidad determinante sobre la conciencia de las estructuras objetivas externas (socioeconómicas, en el caso del primero, o culturales, en lo que se refiere al segundo), participaron también, en mayor o menor grado según sus respectivas corrientes, de esa generalizada conciencia de la modernidad tendente a percibir a los sujetos como artífices conscientes de la marcha de la sociedad y de la historia. Específicamente, en el marxismo dos casos muy significativos a este respecto son los de Gramsci y Lukács. En cuanto, al estructural-funcionalismo, cabe mencionar a Parsons, quien, en su primera obra de importancia, La estructura de la acción social (1968), publicada inicialmente en 1937, consideraba que el camino acertado consiste en concebir la estructura social de un modo que no se vean amenazadas la subjetividad y la libertad. Su finalidad era plantear una especie de estructuralismo voluntarista de acuerdo con el cual postulaba un cierto grado de protagonismo o voluntarismo de los actores sociales en su relación con las condiciones inalterables y coercitivas que estructuralmente se les oponen. Esta orientación voluntarista a la hora de concebir la estructura social fue relegada por Parsons cuando, en un período ulterior del desarrollo de su obra, evolucionó hacia un enfoque netamente estructural-funcionalista. Así sucedió en 1951 cuando publicó El sistema social, obra en la que se manifiesta una marcada tendencia a considerar a los actores sociales como simples agentes o portadores de un entramado estructural abstracto, o de un sistema activado desde fuera por la fuerza de los valores.
} 
considerar o a ser desechados, así como los contenidos específicos de que han de ser dotados están, obviamente, en función de la naturaleza y las circunstancias particulares de la situación a estudiar.

a) Dimensión socioeconómica. En primer lugar, los procesos de incremento de la competitividad y de trasnacionalización, que experimentan los actores empresariales o socioeconómicos en el seno de las estructuras microsociales en las que están insertos, pueden ser interpretados como reacciones reflexivas que,

Tabla 1.

Dimensiones de los procesos a considerar en un análisis sistémico de las estructuras sociales en el marco de la globalización*

\begin{tabular}{|c|c|c|}
\hline $\begin{array}{l}\text { DIMENSIONES } \\
\text { OBJETIVAS }\end{array}$ & $\begin{array}{l}\text { PROCESOS AUTOPOIÉTICOS } \\
\text { A ESCALA MACROSOCIAL O } \\
\text { GLOBAL }\end{array}$ & $\begin{array}{l}\text { PROCESOS REFLEXIVOS A } \\
\text { ESCALA MICROSOCIAL O } \\
\text { LOCAL }\end{array}$ \\
\hline SOCIOECONÓMICA & $\begin{array}{l}\text { - Avance tecnológico } \\
\text { - Globalización económica } \\
\text { - Desregulación y crisis } \\
\text { - Desintegración y fragmentación } \\
\text { social } \\
\text { - Desterritorialización }\end{array}$ & $\begin{array}{l}\text { - Competitividad } \\
\text { - Trasnacionalización } \\
\text { - Búsqueda del desarrollo local } \\
\text { - Reemergencia de lo grupal o } \\
\text { comunitario } \\
\text { - Reterritorialización }\end{array}$ \\
\hline $\begin{array}{l}\text { POLITICO- } \\
\text { INSTITUCIONAL }\end{array}$ & $\begin{array}{l}\text { - Instituciones u organizaciones } \\
\text { supranacionales } \\
\text { - Burocratización y corporativismo }\end{array}$ & $\begin{array}{l}\text { - Reemergencia de poderes locales } \\
\text { o regionales } \\
\text { - Nuevos movimientos sociales }\end{array}$ \\
\hline $\begin{array}{l}\text { DIMENSIÓN } \\
\text { SUBJETIVA }\end{array}$ & $\begin{array}{l}\text { PROCESOS AUTOPOIÉTICOS } \\
\text { A ESCALA MACROSOCIAL O } \\
\text { GLOBAL }\end{array}$ & $\begin{array}{l}\text { PROCESOS REFLEXIVOS A } \\
\text { ESCALA MICROSOCIAL O } \\
\text { LOCAL }\end{array}$ \\
\hline $\begin{array}{l}\text { SIMBÓLICO- } \\
\text { LEGITIMADORA }\end{array}$ & $\begin{array}{l}\text { - Pluralismo relativista } \\
\text { - Crisis paradigmática } \\
\text { - Creciente homogeneización } \\
\text { sociocultural } \\
\text { - Universalismo }\end{array}$ & $\begin{array}{l}\text { - Tolerancia } \\
\text { - Fundamentalismos } \\
\text { - Aumento de la heterogeneidad, } \\
\text { Estilos de vida } \\
\text { - Particularismos }\end{array}$ \\
\hline
\end{tabular}

Fuente: Elaboración propia.

* Aunque soy plenamente consciente de las dificultades de condensar en una tabla de este tipo los complejos procesos que en ella se refieren, sobre todo porque se corre el riesgo de presentar una visión demasiado esquemática y reduccionista de ellos, he optado, no obstante, por elaborar dicha tabla con la pretensión de que en ella el lector pueda ver de, de manera sintética e interrelacionada, los procesos sobre los que después se reflexiona más detalladamente. 


\section{RIS}

ante los procesos económicos y de avance tecnológico que se producen a escala macrosocial global, tienden a propiciar el crecimiento económico o, incluso a veces, a asegurar el mantenimiento del status quo de esos actores.

Asimismo, frente a los procesos globales de desregulación socioeconómica y a la sensación generalizada de heteronomía y de crisis que éstos conllevan para los actores insertos en las estructuras sociales concretas a escala microsocial o local, tienden a afirmarse las presentes tendencias hacia la búsqueda del desarrollo de los entornos locales. Estas tendencias pueden ser consideradas como la expresión de procesos reflexivos a nivel microsocial que, independientemente de sus fines explícitos o conscientes, se encaminan a generar fórmulas de desarrollo protagonizadas desde y por los actores individuales y colectivos insertos en las estructuras sociales locales. A su vez, en el seno de éstas están siendo desplazadas, a menudo, las solidaridades y antagonismos de clase determinantes tradicionalmente de su conformación y dinámica como consecuencia de que están experimentando procesos reflexivos o adaptativos ante la creciente fragmentación, diferenciación y diversificación de la estructura de clases que está teniendo lugar (Alonso, 1992: 134). Una consecuencia de esto es la reemergencia de vinculaciones grupales o comunitarias, en las que la motivación territorial destaca sobre la inherente a la posición de clase. Las tendencias al fortalecimiento de esas vinculaciones, que en principio eran características del mundo tradicional, suelen constituir, también, respuestas de las comunidades locales frente a los riesgos ambientales o los problemas socioeconómicos que para su territorio se derivan de determinadas directrices o políticas fijadas, generalmente, a bastante distancia y con efectos de alcance global. En estos casos, en los que las consecuencias de la globalización son sentidas en mayor o menor medida por la totalidad de habitantes de esas comunidades, tienden a ser dejadas de lado las discrepancias o antagonismos de clase, a la vez que emergen unas nuevas formas de solidaridad consistentes en la articulación conjunta de movimientos sociales interclasistas de protesta o de reivindicación.

La globalización conlleva, asimismo, procesos de gradual desterritorialización de las estructuras sociales de los entornos locales concretos. Desterritorialización significa aquí que la conformación y la dinámica de dichas estructuras tienden a estar condicionadas, cada vez más, por acuerdos e intereses tramados en ámbitos socioespaciales ajenos y, por lo general, bastante distantes. En otras palabras, los procesos de producción y reproducción de las estructuras sociales locales han disminuido su grado de vinculación con el territorio en el que se desarrollan, vinculación que solía ser bastante alta en el mundo tradicional. Frente a estos procesos de desterritorialización tienden a afirmarse procesos de reterritorialiżación. Éstos pueden ser interpretados como reacciones reflexivas de los actores integrantes de las estructuras microsociales locales ante los procesos que inciden sobre ellas a escala global o macroestructural. Con relativa frecuencia, 
estos procesos encaminados a la reterritorialización no se quedan sólo en la búsqueda del desarrollo local, en la razonable reivindicación de la identidad y de la autoctonía local o en reivindicaciones de mayor capacidad de maniobra, sino que, incluso, llegan a elaborar ideologías o a articular movimientos sociales imbuidos de un nacionalismo más o menos violento, excluyente y dogmático. La considerable extensión de estos fenómenos y el radicalismo que, a veces, manifiestan pueden ser interpretados como la expresión de actitudes reflexivas de particularismo y de rechazo visceral a las tendencias universalizadoras de la globalización. Como Robertson (1993, cap. 6) ha destacado, se está experimentando en nuestro tiempo una especie de extensión o universalización del particularismo. Cuando esto tiene lugar de manera extremista o "fundamentalista", las tentativas de territorialización apuntan hacia el aislamiento y exclusión de las estructuras sociales locales, hacia la negación de su ineludible vinculación con lo global, lo que, si bien puede suscitar la idea de que aumenta el grado de autonomía y territorialización de tales estructuras, en realidad suele contribuir a dificultar las posibilidades de progreso y de desarrollo en el seno de las mismas, que son más fáciles de lograr cuando, en vez de tratar de cerrarse al hecho de la globalización, se procura buscar fórmulas o cauces para relacionarse más ventajosamente con ella.

b) Dimensión politico-institucional. En el presente contexto de creciente globalización tienden a articularse nuevas formas de la dimensión políticoinstitucional de las estructuras sociales. En gran medida, esto se debe a que la capacidad y el margen de maniobra de los Estados están siendo erosionados tanto por arriba como por abajo (Entrena, 1998). Por arriba, a causa de la gradual extensión de instituciones u organizaciones supranacionales, y por abajo, a escala microsocial o local, porque, en una situación en la que a raíz de la globalización muchas regiones han entrado en crisis, como reacción reflexiva frente a ello, tiende a producirse una reemergencia o afirmación de los poderes de los territorios locales o regionales. Éstos están reclamando mayores competencias o aumentando éstas, lo que, a su vez, contribuye a incrementar sus posibilidades en lo relativo a acrecentar su grado de control sobre la gestión de los procesos que determinan la producción y reproducción de sus estructuras sociales. En el seno de tales territorios tienden a surgir o a fortalecerse, también, nuevos movimientos sociales que, desde la base de la sociedad, tratan de articular formas de solidaridad social más abiertas, vivas y dinámicas que las que propician las, más o menos centralizadas, corporativas, impenetrables y rígidas, estructuras burocráticas de los partidos políticos o de las grandes organizaciones o corporaciones supranacionales dominantes en el globalizado mundo actual. Como directriz general para entender estos nuevos movimientos sociales, puede señalarse que en ellos, más que las razones de naturaleza clasista o ideológica, tienden a ser determinantes esas identificaciones de índole territorial y comunitaria a las que he hecho referencia antes. 


\section{RIS}

c) Dimensión simbólico-legitimadora. Los procesos contemplados en esta tercera dimensión son los propiamente subjetivos, ya que a través de ellos los sujetos tratan de legitimar, a nivel microsocial o local, la situación macrosocial global en la que están y se sienten insertos, es decir, intentan explicar y/o justificar esa situación, hacer que sea simbólicamente significativa para ellos, que tenga sentido. No obstante, no es mi pretensión diferenciar $u$ oponer claramente lo objetivo y lo subjetivo, pues soy consciente de que los distintos procesos contemplados en cada una de las dimensiones de este modelo, funcionan y son percibidas como realidades objetivas o subjetivas según los casos y circunstancias. En otros términos, tales procesos constituyen hechos objetivos externos cuya experimentación sólo es posible en tanto que son interiorizados subjetivamente por los sujetos concretos, los que, a la vez que están condicionados por ellos, contribuyen en mayor o menor grado a modificarlos o a reproducirlos.

También, los procesos incluidos a escala microsocial en esta dimensión se manifiestan como reacciones reflexivas frente a sus equivalentes a nivel macrosocial. Así, la tolerancia es una de las posibles reacciones valorativo-culturales que se suscitan ante las tendencias globales hacia la generalización de un pluralismo de naturaleza relativista. A su vez, como procesos de reacción reflexiva visceral frente a las incertidumbres y crisis paradigmáticas derivadas de la extensión del relativismo a escala planetaria, tienden a originarse o a radicalizarse los fundamentalismos. Por otra parte, los procesos encaminados al aumento de la heterogeneidad y a la afirmación de lo que se ha dado en llamar estilos de vida, a escala microsocial, pueden ser interpretados como intentos de búsqueda de la autorealización, la singularidad o la autenticidad en un marco global de creciente homogeneización sociocultural ${ }^{12}$. Ésta conlleva un progresivo incremento del universalismo frente al que, de manera complementaria, tienden a fortalecerse los particularismos a escala microsocial o local como reacción reflexiva.

\section{AUTOPOIESIS A NIVEL MACROSOCIAL VERSUS REFLEXIVIDAD A ESCALA MICROSOCIAL}

Como nos recuerda Dorothy E. Smith (1987), las estructuras sociales no pueden entenderse separadamente del mundo de la vida cotidiana. Pues bien, muchos de los procesos que determinan la estructura microsocial de dicho mundo han perdido, definitivamente, su carácter localista. A nivel microsocial,

${ }^{12} \mathrm{Me}$ baso para hacer estas observaciones en un seguimiento no literal de Pérez de Guzmám (1998:9). 
como ya se ha dicho antes, la actividad socio-vital diaria de las personas sigue en su mayor parte teniendo lugar en ámbitos localizables espacial o socialmente. No obstante, tanto en el caso de las microestructuras de interacción de estas redes relacionales de carácter transnacional, crecientemente extendidas en nuestro tiempo, en las que se desenvuelve una significativa parte de población, como cuando se trata de las microestructuras ubicadas en espacios locales concretos, las acciones sociales están cada vez más conectadas con lo que sucede a escala global e influenciadas por ello.

A escala macrosocial o global, los procesos determinantes de la conformación y la dinámica de las estructuras sociales se manifiestan como autopoiéticos. Digo esto en el sentido de que puede afirmarse que dichos procesos son autoreferentes; es decir, operan de acuerdo con unos códigos propios cuya lógica de funcionamiento se desarrolla al margen de la voluntad, los intereses, las finalidades y las intenciones de la inmensa mayoría de los sujetos individuales y colectivos en ellos inmersos. Éstos, como consecuencia de ello, ven profundamente afectado el ámbito de interacción micro de las estructuras sociales en las que transcurre su vida diaria. Sin embargo, también puede considerarse que dichos sujetos son, en cierto modo y en mayor o menor medida según los casos, protagonistas o artífices de los procesos que dan lugar a la globalización, la cual, desde este punto de vista, es susceptible de ser percibida como el resultado de una serie de acciones humanas pasadas y presentes que se han desarrollado 0 desarrollan en el seno de las estructuras microsociales de la cotidianeidad y que, desde la economía, la política, la ciencia o la cultura, han contribuido o contribuyen a generar o a reproducir tales procesos ${ }^{13}$. Pero, esto no quiere decir que la globalización sea la expresión de los propósitos conscientes o explícitos

\footnotetext{
${ }^{13}$ Como habrá notado el lector, entre la afirmación de que los procesos que ocasionan la globalización son autopoiéticos y la de que la globalización es el resultado de acciones humanas hay una cierta contradicción. Es, precisamente, esa contradicción la que se trata de reflejar en este epígrafe. A este respecto, deseo aclarar que, al usar aquí el concepto de autopoiesis, no he seguido literalmente a Niklas Luhmann, sólo me he inspirado en él. Como es sabido, Luhmann pretendió hacer una sociología radicalmente sistémica en la que ya no tenían cabida los viejos conceptos ilustrados, más o menos idealistas, de voluntad, finalidad o racionalidad consciente de la acción de los sujetos que habían servido de base a autores como Weber o Parsons (Ibáñez, 1992: 24). La idea central de Luhmann, según Izuzquiza (1990), era la de «una sociedad sin hombres», una sociedad que constituye un sistema de procesos autopoiéticos o autoreferentes que se desarrollan de acuerdo con su propia lógica y códigos intrínsecos, al margen de la voluntad o intenciones conscientes de los sujetos individuales y colectivos en ellos inmersos (Luhmann, 1990). En esta sociedad los sujetos son meros entornos externos a los sistemas sociales, cuya acción constituye un elemento que sólo puede ser explicado «desde arriba»; es decir, desde la lógica estructural del sistema que
} 
de los sujetos humanos a raíz de cuya actuación se ha producido o reproduce, sino sólo un efecto no intencional de las reacciones reflexivas y creativas de los actores sociales insertos en ámbitos locales o relacionales microsociales específicos, sobre los que, a su vez, incide.

Como reacciones reflexivas frente a la globalización, he interpretado los diferentes procesos que se desarrollan a nivel microsocial o local contemplados en la tercera columna de la tabla 1 . Las dimensiones objetivas y subjetivas de tales procesos no hacen referencia sólo a las reacciones reflexivas de la base de la sociedad, sino también a las procedentes de los actores situados en los niveles sociales más altos. La notable influencia y capacidad de decisión de los segundos, cuya acción se desarrolla frecuentemente a través de microestructuras relacionales localizables socialmente pero de alcance transnacional, les sitúan en unas condiciones más propicias para que pueda suscitarse la idea de que son los sujetos o protagonistas sociales de la globalización, la cual, más que como una serie de procesos autopoiéticos, se les manifiesta como resultado de procesos, en gran parte, impulsados y dotados de contenido por ellos. Falaz idea, pues, obviamente, nadie posee el control absoluto de los procesos sociales, y menos aún de los que acarrea la globalización. No obstante, donde la autopoiesis de lo macrosocial o global se manifiesta con más fuerza es en el nivel en el que se desenvuelve la mayoría de la base de la sociedad. Esto, porque la población de este nivel suele estar más predispuesta a sentirse impotente ante la globalización, en gran medida, debido a que la experimenta como un conjunto de procesos al margen de su voluntad, a cuyas exigencias en lo socioeconómico, lo políticoinstitucional y lo simbólico-legitimador se ve, ineludiblemente, impelida a adaptarse. Pero, incluso en este caso, puede observarse una apreciable dosis de creatividad en los efectos transformadores que sobre las estructuras microsociales o locales suelen tener los procesos reflexivos desarrollados por sus actores

\footnotetext{
hace posible su existencia. Esto llevó a dicho autor a negar que el sistema se genere o emerja a partir de la acción y a considerar, al contrario, a la acción como uno de los elementos del sistema, y éstos, según sus propias palabras, «son elementos sólo para los sistemas -ellos los utilizan como unidad-y sólo dentro de ellos llegan a existir, lo cual él formuló con el "concepto de autopoiesis" (1991: 152-153). Pero, yo concibo aquí la autopoiesis de manera diferente a Luhmann. En este trabajo, la autopoiesis no es pensada en el sentido de que sea una condición inherente al sistema social, sino que sólo se afirma que la lógica mediante la que opera la globalización a escala macrosocial sería susceptible de ser considerada como autoreferente. Ello, en contraste con el carácter reflexivo de la acción de los sujetos sociales humanos que se desenvuelven a escala microsocial. Una reflexividad que yo entiendo en el sentido de que la sociedad está compuesta por hombres; es decir, por sujetos con intenciones y voluntades, independientemente de que, con frecuencia, no consigan los resultados esperados o deseados por los que actúan.
} 
sociales frente a la globalización. De ahí, que, como hemos visto en el epígrafe anterior, cada uno de los procesos que acaecen a nivel macrosocial o global tenga su correlato reflexivo en los que suceden a escala microsocial o local. Existe pues, una relación dialéctica de mutua interinfluencia y complementariedad entre unos y otros procesos.

\section{CONCLUSIONES}

Las estructuras sociales ya no pueden ser vistas como unas realidades relativamente autárquicas y autónomas, tal y como era posible concebirlas cuando las sociedades tenían un grado de autosuficiencia, a escala regional o estatal, notablemente mayor que el que tienen ahora. Hoy, las estructuras sociales han de ser necesariamente consideradas como unos entramados sociales vinculados a la realidad global de la que forman parte y por la que están cada vez más influidas en su dinámica de funcionamiento y evolución, en sus procesos de producción y reproducción social. Como consecuencia de la cada vez mayor globalización de tales procesos, suelen experimentarse dos fenómenos opuestos en las actuales estructuras sociales que fluctúan entre las tendencias que apuntan hacia su creciente homogeneización e integración mundial, de una parte, y, de otra, las que se dirigen hacia la búsqueda de su diferenciación y singularidad, dentro las que cabe mencionar las presentes propensiones a reinventar las tradiciones o a reforzar las «identidades» en el seno de determinadas estructuras sociales locales. Si centramos nuestra atención en las primeras tendencias, la globalización podría ser conceptuada como «el conjunto de procesos que conducen a un mundo único» (Robertson, 1992: 396.). Se produce así una especie de unificación mundial que amenaza con vaciar de contenido los contextos de acción de las estructuras sociales a escala local (regional o estatal), cuyos problemas socioeconómicos y procesos de producción y reproducción social se hallan cada vez más vinculados a la imprevisible dinámica globalizadora y suelen desarrollarse en consonancia con intereses y decisiones de alcance mundial tomadas a nivel extralocal ${ }^{14}$. Los efectos desterritorializadores de la globalización sobre las estructuras sociales concretas, que como hemos visto en su mayoría se forman y operan en ámbitos específicos localizables social y espacialmente, se manifiestan en una creciente reducción de las posibilidades de control de su propio funcionamiento y gestión, así como en que su significado social e histórico —es decir, lo que podría conceptuarse como su autoctonía-

${ }^{14}$ Al hacer este razonamiento me baso en Alessandro Bonanno y Karen Bradley (1994, 63). 


\section{RIS}

tienden a ser disociados de su papel económico y de la función que, en general, desempeñan en el actual contexto global ${ }^{15}$. Esta es una de las principales razones del alto grado de complejidad manifestado por las crecientemente globalizadas estructuras de las sociedades actuales, el cual contribuye sobremanera a aumentar las dificultades de descripción y de explicación de las mismas. Dificultades que, sin duda, están en el origen de la crisis existencial y de paradigmas en las Ciencias sociales que se observa en el presente, la cual se manifiesta con especial intensidad en esa heterogénea corriente de pensamiento que se ha dado en llamar posmodernidad ${ }^{16}$.

Ante esta situación, a diferencia del nihilismo y de la visión apocalíptica en que suelen caer los posmodernos, en este trabajo he tratado de racionalizar y explicar la presente complejidad, pues creo que la crisis y las dificultades de las sociedades actuales, así como sus incertidumbres y riesgos ${ }^{17}$, resultan más comprensibles si se los ubica en el contexto de los efectos (en las dimensiones socioeconómica, político-institucional y simbólico-cultural) que sobre los procesos de producción y reproducción de las estructuras sociales acarrea la

\footnotetext{
${ }^{15}$ Me ha sugerido estas argumentaciones la lectura de Manuel Castells $(1987,58)$.

${ }^{16}$ Según autores como Lipovetsky o Vattimo, en la posmodernidad hemos entrado en una nueva era de la humanidad. En esta situación, "se disuelven la confianza y la fe en el futuro, ya nadie cree en el porvenir de la Revolución y el progreso, la gente desea vivir el 'aquí' y 'ahora', buscando la calidad de vida, y la cultura personalizada. La atención por lo social se vuelve hacia el individuo y se difunde el narcicismo individual y corporativo. El individuo sólo tiene ojos para sí mismo o para su grupo" (Picó, 1988: 37).

${ }^{17}$ Según Beck, Giddens y Lash (1995), que no manifiestan esas visiones apocalípticas de los posmodernos, el actual estado de cosas mundial es la manifestación de la victoria del capitalismo, que ha evolucionado y producido nuevas formas sociales. Éstas son el resultado del proceso de desarrollo de la propia modernidad, que ha dado lugar a una radicalización de la misma que ha roto los contornos y premisas en que se sustentaba la sociedad industrial y ha abierto caminos hacia otra modernidad (Beck, 1995: 3). En otros términos, las actuales circunstancias de indeterminación, relativismo e incertidumbre pueden ser consideradas, en realidad, como una consecuencia no intencional ni prevista de las continuas rupturas y autocríticas llevadas a cabo por los sujetos individuales y colectivos, a partir de cuya acción se ha derivado la construcción y evolución de la modernidad. En concreto, Ulrich Beck(1992) ha denominado la época actual como una sociedad del riesgo, ya que en ella, frente a los riesgos e imprevistos clásicos (quiebras, catástrofes, guerras, etc.) sujetos a una previsión razonable, surgen nuevos riesgos que no son fácilmente calculables ni imputables a nadie, y que, además, abren un horizonte de daños irreparables (un caso paradigmático es el accidente nuclear de Chernobyl). En estas circunstancias, no se trata de que ya no sepamos como acertar, sino de que ni siquiera podemos prever con exactitud en que medida nos equivocaremos. Por su parte, Giddens (1996: 13 y 85) se ha referido a la presente situación como una era de incertidumbre fabricada, ya que, en gran medida, es el resultado de la propia acción humana.
} 
globalización. He adoptado esa actitud porque considero que los problemas y la especial complejidad del presente no deben servirnos como excusa para renunciar al desafiante reto de contribuir al desarrollo de herramientas conceptuales encaminadas a sistematizar y a analizar la realidad social de cara a su conocimiento y a la intervención sobre ella. Al fin y al cabo, todas las épocas anteriores también debieron resultar especialmente difíciles de entender para los que las vivieron o trataron de teorizar acerca de las mismas. Especialmente, frente a la crisis paradigmática de las Ciencias sociales actuales, frente a esa extendida tendencia a la fragmentación cognoscitiva o a optar por la "seguridad" que ofrece el estudio de lo fácilmente acotable, en gran parte como prevenciones frente a las simplificaciones en que solían caer los "grandes relatos" $\tan$ característicos de los saberes de la modernidad ${ }^{18}$, en nuestros días se hace especialmente necesario arriesgarse en la exploración de lo difícil y complejo, buscar explicaciones holistas de la realidad social que nos permitan una comprensión totalizante de las cada vez más globalizadas situaciones y problemáticas sociales. Es en este sentido que aquí he tratado de describir y explicar los procesos de producción y reproducción de las estructuras sociales de acuerdo con el modelo antes propuesto. Procesos que, como hemos visto, manifiestan la naturaleza reflexiva e histórica de tales estructuras, así como su relación dialéctica -en tanto que entidades espacial y/o socialmente localizables - con la crecientemente globalizada realidad socioeconómica en la que están cada vez más insertas. Una realidad, cuya lógica de funcionamiento suele mostrarse para la mayoría de población inserta en las estructuras sociales locales concretas como al margen de sus posibilidades de control y de sus intereses e intenciones; de ahí, que haya optado por calificarla como una realidad autopoiética.

\footnotetext{
${ }^{18}$ Según Lyotard, los "grandes relatos" son aquellas narraciones que se refieren en todas las culturas y que cumplen el papel de proporcionar una visión integrada y coherente de los distintos aspectos de la realidad. Desempeñan, por consiguiente, múltiples funciones, tales como: dar cohesión al grupo, legitimar valores y proyectos, o hacer aceptables las normas que regulan una sociedad. En este sentido, pueden ser considerados como "grandes relatos" o "metarrelatos", de la modernidad, por ejemplo, la idea de la emancipación progresiva de la razón humana característica de la modernidad o el concepto de la libertad arraigado en este periodo histórico (Lyotard, 1978: 29-32).
} 


\section{BIBLIOGRAFÍA}

ALONSO, L.E. (1992), «Postfordismo, fragmentación social y crisis de los nuevos movimientos sociales», Sociologia del Trabajo, Nueva época, no 16, pp. 119-141.

BECK, U. (1992), Risk Society. Towards a New Modernity, Sage Publications, London.

BECK, U., A. GIDDENS y S. LASH (1995), Reflexive modernization, Polity Press, Cambridge. Véanse especialmente los capítulos 1 y 4.

BELL, D. (1977), Las contradicciones culturales del capitalismo, Alianza Universidad, Madrid.

BERGER, P.L. (1989), La revolución capitalista. Cincuenta proposiciones sobre la prosperidad, la igualdad y la libertad, Edit. Península, Barcelona.

BERIAIN, J. (comp.) (1996), Las consecuencias perversas de la modernidad: modernidad, contigencia y riesgo, Edit. Anthropos, Barcelona.

BONANNO, A. (1994), "Globalización de sector agrícola y alimentario: crisis de convergencia contradictoria», en A. Bonanno (Comp.). La globalización del sector agroalimentario, Edit. Ministerio de Agricultura Pesca y Alimentación, Serie Estudios, Madrid, pp. 15-49.

BONANNO, A. y K. BRADLEY (1994), «Spatial Relations in the Global Socio-Economic System and the Implications for Development Planning", in David Symes and Anton J. Jansen, eds., Agricultural Restructuring and Rural Change in Europe, Edit. Agricultural University Wageningen, The Netherlands, pp. 49-64.

CASTELLS, M. (1987), "Technological change, economic restructuring and the spacial division of labour», in H. Muegge, Walter B. Stöhr, P. Hesp and B. Stuckey, eds., International Economic Restructuring and the Regional Community, Edit. Avebury, Gran Bretaña, pp. 45-63.

ELIAS, N. (1990), La sociedad y los individuos, Edit. Península, Barcelona.

ENTRENA, F. (1998), "From the credibility crisis of formal organizations to the re-emergence of the group: an ecosystemic approach", Free Inquiry in Creative Sociology, $\mathrm{n}^{\circ} 26$, vol. 1 , Oklahoma State University, USA, pp. 43-54.

GIDDENS, A. (1991), Modernity and Self-Identity: Self and Society in the Late Modern Age, Edit. Stanford University Press, Stanford.

(1993), La estructura de clases en las sociedades avanzadas, Alianza Universidad, Madrid.

(1996), Más allá de la izquierda y la derecha. El futuro de las politicas radicales, Ediciones Cátedra, Madrid.

(1999), Las consecuencias de la modernidad, Edit. Alianza Editorial, Madrid. 
IBÁÑEZ, J. (1979), Más allá de la Sociología. El gnupo de discusión, técnica y crítica, Siglo XXI editores, Madrid.

(1992), "El papel del sujeto en la teoría (Hacia una Sociología reflexiva)", Texto de la ponencia presentada al IV Congreso Español de Sociología, Madrid, 24-26 de Septiembre de 1992.

IZUZQUIZA, I. (1990), La sociedad sin hombres. Niklas Luhmann o la teoria como escándalo, Edit. Anthropos, Barcelona.

LAMO DE ESPINOSA, E. (1990), La sociedad reflexiva. Sujeto y objeto del conocimiento sociológico, Edit. CIS / Siglo Veintiuno Editores, Madrid.

LEVI-STRAUSS, C. (1958), Anthropologie structurale, Plon, París.

LUHMANN, N. (1990), Sociedad y sistema, Edit. Paidos, Buenos Aires.

(1991), Sistemas sociales, Edit. Alianza-Universidad/Iberoamericana, México.

LYOTARD, J. F. (1978), La postmodernidad (explicada para niños), Edit. Gedisa, Barcelona.

MERTON, R. K. (1970), Teoria y estructura sociales, Edit. F.C.E., Mexico.

MILLET, L. y M. VARIN D'AINVELLE (1975), El estructuralismo como método, Editorial Laia, Barcelona.

NADEL, S. F. (1966), Teoria de la estructura social, Edit. Guadarrama, Madrid.

NISBET, R. A. (1975), Introducción a la Sociologia, Edit. Vicens-Vives, Barcelona.

PARSONS, T. (1968), La estructura de la acción social, Edit. Guadarrama, Madrid.

(1976), El sistema social, Edit. Revista de Occidente, Madrid.

PÉREZ DE GUZMÁN, T. (1995), "La sociedad reflejada”, Revista Española de Investigaciones Sociológicas (REIS), $\mathrm{n}^{\circ}$ 69, enero-marzo, pp. 175-201.

(1998), "Dialécticas de identidad y exclusión en la modernidad avanzada", Texto de la ponencia presentada al I Congreso sobre Exclusión Social en los Países Ibéricos, Lisboa, marzo de 1998.

PICÓ, J. (1988), Modernidad y posmodernidad, Alianza Editorial, Madrid.

RICOEUR, P. (1969), Finitud y culpabilidad, Edit. Taurus, Madrid.

ROBERTSON, R. (1992), "Globality, global culture and images of world order”, en Haferkamp y Smelser, eds., Social Change and Modernity, Edit. Berkeley, University of California Press, pp. 395-411.

(1993), Globalization. Social Theory and Global Culture, SAGE Publications, London. 


\title{
RIS
}

REVISTA INTEANACIONAL DE SOCIOLOGIA

№ 27, Septiembre - Diciembre, 2000

FRANCISCO ENTRENA IUURÁN

SMITH, D. E. (1987), The Everyday World as Problematic: A feminist Sociology, Edit. Northeastern University Press, Boston.

SZTOMPKA, P. (1995), Sociologia del cambio social, Edit. Alianza Editorial, Madrid.

WORSLEY, P. (1984), The Three Worlds: Culture and World Development, Edit. Weidenfeld and Nicolson, Londres.

\begin{abstract}
Social structures are considered in this essay as socially built realities that are subjected to reflexivity and historicity and whose production and reproduction are increasingly inserted in the globalization processes that nowadays are taking place at a planetary scale. As a consequence of this situation, a remarkable intensification of reflexivity in social structures is being experienced, and consequently we are seeing a progressive increasing on their complexity level. Besides the task of describing and /or explaining them becomes harder. With the purpose of carrying out this task from a holist and totalizing approach, in this article some analytic criteria are proposed whose theoretical framework intends to be suitable for the study of the dialectic relations which are experienced between micro and macro. On the one hand, what is conceived as reflexive nature of the micro-social structures in those most people daily life is developed; and, on the other hand, the logic, considered here as autopoietic or autoreferential, through which the macro-social structures perform at a global scale.
\end{abstract}

E-mail: fentrena@goliat.ugr.es 